\title{
A UNIFYING FRAMEWORK FOR GENERALIZATIONS OF THE ENESTRÖM-KAKEYA THEOREM
}

\author{
A. Melman \\ Department of Applied Mathematics \\ School of Engineering, Santa Clara University \\ Santa Clara, CA 95053 \\ e-mail : amelman@scu.edu
}

\begin{abstract}
The classical Eneström-Kakeya theorem establishes upper and lower bounds on the zeros of a polynomial with positive coefficients that are explicit functions of those coefficients. We establish a unifying framework that incorporates this theorem and several similar ones as special cases, while generating new theorems of a similar type. These establish zero inclusion and exclusion regions consisting of a single disk or the union of several disks in the complex plane. Our framework is built on two basic tools, namely a generalization of an observation by Cauchy, and a family of polynomial multipliers. Its approach is transparent and reduces algebraic manipulations to a minimum.
\end{abstract}

Key words : polynomial, positive coefficients, Cauchy, Eneström-Kakeya

AMS(MOS) subject classification : 12D10, 30C15, 65H05

\section{1 introduction}

The Eneström-Kakeya theorem ([6], [7, and [10]) establishes upper and lower bounds on the moduli of the zeros of a polynomial with positive coefficients that are simple explicit functions of the coefficients. It has been extended and generalized in different ways, e.g., by relaxing the restrictions on the coefficients, or by deriving different regions of the complex plane that also contain all the zeros of the given polynomial. Sharpness of the bounds was considered in 1 1, 2, and 9. A good overview of these generalizations can be found in [8] and for additional historical remarks about this pretty theorem we also refer to [16, p. 271-272].

Here we establish a framework that generates theorems similar to that of Eneström and Kakeya, namely, theorems that derive regions in the complex plane that contain or do not contain the zeros of polynomials with positive coefficients. These regions, which consist of a single disk or of the union of several disks, are explicitly determined, i.e., they do not require numerical methods. We obtain our results by using the same two basic tools for all of them: a family of polynomial multipliers and a generalization of an observation by Cauchy. This transparent approach reduces algebraic manipulations to a minimum, unifies the derivation of these results, and generates new ones while incorporating the EneströmKakeya theorem and some of its variants as special cases. Some of the regions derived in this way consist of several disks, which can provide additional information about the zeros. Regions of this kind have not been considered in any of the existing generalizations of the Eneström-Kakeya theorem. 
The paper is organized as follows. We begin in Section 2 by collecting a few results that are needed further on. In Section 3, we derive zero inclusion and exclusion regions composed of a single disk centered at the origin, while in Section 4 we obtain disks that are not centered at the origin. In Sections 5 and 6, we derive zero inclusion regions consisting of two and three disks, respectively. The appendix contains a technical lemma needed in Section 4 .

\section{Preliminaries}

In this section, we collect a few theorems and definitions that will be needed further on. Throughout this section, we consider a polynomial $p(z)=a_{n} z^{n}+a_{n-1} z^{n-1}+\cdots+a_{1} z+a_{0}$ with complex coefficients.

Definition 2.1. The Cauchy radius of the $k$ th kind of $p$ is defined as the unique positive solution $s_{k}$ of

$$
\left|a_{n}\right| z^{n}+\left|a_{n-1}\right| z^{n-1}+\cdots+\left|a_{n-k+1}\right| z^{n-k+1}-\left|a_{n-k}\right| z^{n-k}-\cdots-\left|a_{1}\right| z-\left|a_{0}\right|=0 .
$$

When $k=1, s_{1}$ is simply called the Cauchy radius of $p$.

The following theorem is Theorem 3.2 in [13], where $k$ here corresponds to $n-k$ in that theorem.

Theorem 2.1. All the zeros of the complex polynomial $p(z)=\sum_{j=0}^{n} a_{j} z^{j}$ lie in the sets

$$
\begin{aligned}
\Gamma_{1}(k)=\left\{z \in \mathbb{C}: \mid a_{n} z^{k}+a_{n-1} z^{k-1}+\cdots+\right. & a_{n-k+1} z \mid \\
& \left.\leq\left|a_{n}\right| s_{k}^{k}+\left|a_{n-1}\right| s_{k}^{k-1}+\cdots+\left|a_{n-k+1}\right| s_{k}\right\}
\end{aligned}
$$

and

$$
\begin{aligned}
\Gamma_{2}(k)=\left\{z \in \mathbb{C}:\left|a_{n} z^{k}+a_{n-1} z^{k-1}+\cdots+a_{n-k+1} z+a_{n-k}\right|\right. & \\
& \left.\leq\left|a_{n}\right| s_{k+1}^{k}+\left|a_{n-1}\right| s_{k+1}^{k-1}+\cdots+\left|a_{n-k+1}\right| s_{k+1}+\left|a_{n-k}\right|\right\},
\end{aligned}
$$

whose boundaries are lemniscates and where $s_{j}$ is the Cauchy radius of the $j$ th kind, defined in Definition 2.1.

If $\Gamma_{1}(k)$ or $\Gamma_{2}(k)$ consists of disjoint regions whose boundaries are simple closed (Jordan) curves and $\ell$ is the number of foci of its bounding lemniscate contained in any such region, then that region contains $\ell$ zeros of $p$ when it does not contain the origin, and $\ell+n-k$ zeros of $p$ when it does contain the origin.

For the special case $\Gamma_{1}(1)$ in Theorem 2.1, we obtain that all the zeros of $p$ lie in the disk defined by $|z| \leq s_{1}$, where $s_{1}$ is the unique positive solution of

$$
\left|a_{n}\right| z^{n}-\left|a_{n-1}\right| z^{n-1}-\cdots-\left|a_{1}\right| z-\left|a_{0}\right|=0 .
$$


This is a classical observation by Cauchy from 1829 ([5], see also, e.g., [11, Th.(27,1), p.122 and Exercise 1, p.126], [15, Theorem 3.1.1], [16, Theorem 8.1.3]).

In the special case of $\Gamma_{2}(1)$ in Theorem 2.1, we obtain that all the zeros of $p$ lie in the disk defined by $\left|z+a_{n-1} / a_{n}\right| \leq s_{2}+\left|a_{n-1} / a_{n}\right|$, where $s_{2}$ is the unique positive solution of

$$
\left|a_{n}\right| z^{n}+\left|a_{n-1}\right| z^{n-1}-\left|a_{n-2}\right| z^{n-2}-\cdots-\left|a_{1}\right| z-\left|a_{0}\right|=0 .
$$

This is Theorem 1 in $[12$.

The boundaries of $\Gamma_{1}(k)$ and $\Gamma_{2}(k)$ are lemniscates that, as $k$ increases, become too complicated to use for deriving explicit zero inclusion regions. Instead, we will approximate a region $\Gamma$, bounded by a lemniscate of the form $|q(z)|=R$, where $q(z)=z^{m}+b_{m-1} z^{m-1}+$ $\cdots+b_{0}$, as follows. Denoting the zeros of $q$ by $c_{j}$, we have that

$$
|q(z)|=\left|z^{m}+b_{m-1} z^{m-1}+\cdots+b_{0}\right|=\left|z-c_{1}\right|\left|z-c_{2}\right| \ldots\left|z-c_{m}\right|,
$$

so that

$$
\Gamma=\{z \in \mathbb{C}:|q(z)| \leq R\}=\left\{z \in \mathbb{C}:\left|z-c_{1}\right|\left|z-c_{2}\right| \ldots\left|z-c_{m}\right| \leq R\right\}
$$

This means that

$$
\Gamma \subseteq \bigcup_{j=1}^{m}\left\{z \in \mathbb{C}:\left|z-c_{j}\right| \leq R^{1 / m}\right\} .
$$

Although larger than $\Gamma$, this union of disks is easier to work with, and, as we shall see, can still be useful. It may sometimes be better to allow different disks to have different radii, but in the interest of simplicity we will use the same radius for all disks.

Additional zero inclusion regions can be obtained by applying Theorem 2.1 to the reverse polynomial $p^{\#}(\zeta)=a_{0} \zeta^{n}+a_{1} \zeta^{n-1}+\cdots+a_{n-1} \zeta+a_{n}$ (with $a_{0} \neq 0$ ), whose zeros are the reciprocals of the zeros of $p$.

\section{Single disk centered at the origin}

In this section we derive inclusion and exclusion regions consisting of a single disk centered at the origin for polynomials with positive coefficients. We begin by stating the EneströmKakeya theorem ([6], [7], and [10]) and, for completeness, also provide a standard proof.

Theorem 3.1. (Eneström-Kakeya) All the zeros of the real polynomial $p(z)=\sum_{j=0}^{n} a_{j} z^{j}$ with positive coefficients lie in the annulus defined by

$$
\left\{z \in \mathbb{C}: \min _{0 \leq j \leq n-1} \frac{a_{j}}{a_{j+1}} \leq|z| \leq \max _{0 \leq j \leq n-1} \frac{a_{j}}{a_{j+1}}\right\}
$$

Proof. Consider $(z-\gamma) p(z)$, where $\gamma \in \mathbb{R}$ :

$$
(z-\gamma) p(z)=a_{n} z^{n+1}+\sum_{j=1}^{n}\left(a_{j-1}-\gamma a_{j}\right) z^{j}-\gamma a_{0} .
$$


Clearly, any upper bound on the zeros of $(z-\gamma) p(z)$ will also be an upper bound on the zeros of $p$. From (4) we observe that all the coefficients of $(z-\gamma) p(z)$, except that of $z^{n+1}$, will be nonpositive if

$$
\gamma=\max _{0 \leq j \leq n-1} \frac{a_{j}}{a_{j+1}},
$$

while the constant term is negative. For that value of $\gamma$, the Cauchy radius of $(z-\gamma) p(z)$ is the unique positive solution of $(z-\gamma) p(z)=0$ and, since $\gamma>0$ is a positive zero of $(z-\gamma) p(z)$, it must be equal to the Cauchy radius. This proves the upper bound. The lower bound is proved analogously by considering the reverse polynomial of $p$.

The key ingredients in this proof are the multiplier $z-\gamma$ and the Cauchy radius, a pattern that will repeat itself in subsequent results, albeit with different multipliers and Cauchy radii of a higher kind. The use of multipliers is standard practice throughout the literature related to the Eneström-Kakeya theorem.

It is worth pointing out that the Eneström-Kakeya bounds are not necessarily better than those obtained from the Cauchy radii of $p$ and $p^{\#}$, although their obvious advantage is that they are explicit and therefore do not require the solution of a polynomial equation. Consider the following example.

Example. Define the polynomials $p_{1}(z)=2 z^{5}+z^{4}+4 z^{3}+z^{2}+2 z+3$ and $p_{2}(z)=$ $z^{5}+z^{4}+2 z^{3}+3 z^{2}+2 z+1$. For $p_{1}$, whose smallest and largest zeros have magnitudes 0.7740 and 1.3921 , respectively, the Cauchy radii determine an interval of $[0.6288,1.9242]$ for the magnitudes of the zeros, while the Eneström-Kakeya theorem gives [0.25, 4.00], which is worse.

On the other hand, for $p_{2}$, with smallest and largest zero magnitudes 0.7136 and 1.4013 , respectively, we obtain $[0.3143,2.4654]$ and $[0.5,2.0]$ from the Cauchy radii and Eneström-Kakeya, respectively. Here, the Eneström-Kakeya bounds are better.

There is one clear case where the Eneström-Kakeya theorem is guaranteed to produce a better upper bound than the Cauchy radius, namely, when $a_{n-1} / a_{n}=\max _{1 \leq j \leq n} a_{j-1} / a_{j}$. This is an immediate consequence of Theorem 8.3.1 in [16], which states that, if the Cauchy radius of $p$ is not a zero of $p$ (which is obviously the case since all of its coefficients are positive), then the Cauchy radius of $\left(z-a_{n-1} / a_{n}\right) p(z)$, which here is equal to $a_{n-1} / a_{n}$, is strictly smaller than that of $p$ for any complex polynomial $p$, whose coefficients are all nonzero. This can also easily be seen by substituting $a_{n-1} / a_{n}$ in the left-hand side of (2), which yields a negative value, indicating that $a_{n-1} / a_{n}$ is less than the Cauchy radius. Analogously, the Eneström-Kakeya theorem will yield a better lower bound than that obtained from the Cauchy radius if $a_{0} / a_{1}=\min _{1 \leq j \leq n} a_{j-1} / a_{j}$.

We can obtain more results like the Eneström-Kakeya theorem by simply changing the multiplier used in its proof, while using Theorem 2.1 to obtain bounds. The following two theorems do just that.

Theorem 3.2. Let the real polynomial $p(z)=\sum_{j=0}^{n} a_{j} z^{j}$ with $n \geq 2$ have positive coefficients.

(a) Let

$$
\gamma_{1}=\frac{a_{n-1}}{a_{n}} \text { and } \gamma_{0}=\max \left\{0, \max _{0 \leq j \leq n-2} \frac{a_{j}-\gamma_{1} a_{j+1}}{a_{j+2}}\right\}
$$


then all the zeros of $p$ lie in the disk defined by $|z| \leq \frac{1}{2}\left(\gamma_{1}+\left(\gamma_{1}^{2}+4 \gamma_{0}\right)^{1 / 2}\right)$.

(b) Let

$$
\delta_{1}=\frac{a_{1}}{a_{0}} \text { and } \delta_{0}=\max \left\{0, \max _{0 \leq j \leq n-2} \frac{a_{j+2}-\delta_{1} a_{j+1}}{a_{j}}\right\},
$$

then all the zeros of $p$ are excluded from an open disk defined by

$$
|z|<\frac{2}{\delta_{1}+\left(\delta_{1}^{2}+4 \delta_{0}\right)^{1 / 2}} .
$$

Proof. Consider $q(z)=\left(z^{2}-\gamma_{1} z-\gamma_{0}\right) p(z)$ for $\gamma_{0}, \gamma_{1} \in \mathbb{R}$ :

$$
q(z)=a_{n} z^{n+2}+\left(a_{n-1}-\gamma_{1} a_{n}\right) z^{n+1}+\sum_{j=2}^{n}\left(a_{j-2}-\gamma_{1} a_{j-1}-\gamma_{0} a_{j}\right) z^{j}-\gamma_{1} a_{0} z-\gamma_{0} a_{0} .
$$

Any set containing all the zeros of $q$ will also contain all those of $p$. If all coefficients in equation (5), other than the leading one, are nonpositive, then the Cauchy radius of $q$ is its own unique positive zero. With $\gamma_{1}=a_{n-1} / a_{n}$, the smallest such value for $\gamma_{0}$ is given by $\gamma_{0}=\max _{0 \leq j \leq n-2}\left(a_{j-2}-\gamma_{1} a_{j-1}\right) / a_{j}$. If this value is negative, then we must set $\gamma_{0}=0$ to make the constant coefficient in (5) nonpositive. The Cauchy radius is then the unique positive zero of the quadratic multiplier, which is $\frac{1}{2}\left(\gamma_{1}+\left(\gamma_{1}^{2}+4 \gamma_{0}\right)^{1 / 2}\right)$. This proves part (a).

For part (b), we apply the result in part (a) to the reverse polynomial $p^{\#}(\zeta)=a_{0} \zeta^{n}+$ $a_{1} \zeta^{n-1}+\cdots+a_{n-1} \zeta+a_{n}$, whose zeros are the reciprocals of those of $p$. It is a straightforward exercise to replace $a_{j}$ by $a_{n-j}$ in part (a) to obtain the equivalent expressions for the reciprocals $\zeta=1 / z$ of the zeros $z$ of $p$, bearing in mind that $|\zeta| \leq M$ implies that $|z| \geq 1 / M$. This concludes the proof.

Theorem 3.2 delivers a better bound than the Cauchy radius when $\gamma_{0}=\left(a_{n-2}-\right.$ $\left.\gamma_{1} a_{n-1}\right) / a_{n}$. This is a consequence of Theorem 3.1 in [14, which states that, if the Cauchy radius of $p$ is not a zero of $p$, then the Cauchy radius of $\left(z^{2}-\left(a_{n-1} / a_{n}\right) z-a_{n-2} / a_{n}+\right.$ $\left.a_{n-1}^{2} / a_{n}^{2}\right) p(z)$ is strictly smaller than that of $p$. Analogously, we find that Theorem 3.2 yields a better lower bound than can be obtained from the Cauchy radius when $\delta_{0}=$ $\left(a_{2}-\delta_{1} a_{1}\right) / a_{0}$. We also note that, if $a_{n-1} / a_{n}=\max _{0 \leq j \leq n-1} a_{j} / a_{j+1}$, then $\gamma_{0}=0$ and the upper bound from this theorem is identical to the Eneström-Kakeya upper bound, which in this case is smaller (better) than the Cauchy radius, as we saw before. An analogous argument holds for the lower bound.

Although somewhat dissimilar at first sight, part (a) of this theorem is essentially Theorem 1 in 3 , with a few differences. Our result is formulated in terms of parameters that are the coefficients of a multipier, whereas in [3] the parameters are the zeros of the multiplier. This is merely a question of preference, although using the coefficients leads to simpler expressions. In cite [3], the parameters of the multiplier are left to be determined as long as they make the appropriate coefficients nonpositive, exactly like our parameters here, but with the distinction that we actually assign values to the parameters to satisfy that requirement. More importantly, the proof in [3] relies on more complicated arguments 
than the Cauchy radius. That, combined with using zeros rather than coefficients as parameters, would appear to make it more difficult to use higher order multipliers to obtain results as in the next theorem. Finally, although a minor matter, no lower bound was mentioned in [3].

Theorem 3.2 sets the stage for similar results. The following theorem is a prototype for such results and it illustrates the straightforward manner in which they can be obtained. They are only marginally more complicated than Theorem 3.2. but they tend to produce better results, as we will see later.

Theorem 3.3. Let the real polynomial $p(z)=\sum_{j=0}^{n} a_{j} z^{j}$ with $n \geq 3$ have positive coefficients, then the following holds.

(a) If

$$
\gamma_{2}=\max \left\{\frac{a_{n-1}}{a_{n}}, \frac{a_{n-2}}{a_{n-1}}\right\}, \gamma_{1}=0, \gamma_{0}=\max \left\{0, \max _{0 \leq j \leq n-3} \frac{a_{j}-\gamma_{2} a_{j+1}}{a_{j+3}}\right\},
$$

or if

$$
\begin{aligned}
& \gamma_{2}=\frac{a_{n-1}}{a_{n}}, \\
& \gamma_{1}=\frac{a_{n-2}-\gamma_{2} a_{n-1}}{a_{n}}, \\
& \gamma_{0}=\max \left\{0, \frac{\gamma_{1} a_{0}}{-a_{1}}, \frac{\gamma_{2} a_{0}+\gamma_{1} a_{1}}{-a_{2}}, \max _{0 \leq j \leq n-3} \frac{a_{j}-\gamma_{2} a_{j+1}-\gamma_{1} a_{j+2}}{a_{j+3}}\right\},
\end{aligned}
$$

then all the zeros of $p$ lie in the disk defined by $|z| \leq r_{1}$, where $r_{1}$ is the unique positive zero of $z^{3}-\gamma_{2} z^{2}-\gamma_{1} z-\gamma_{0}$.

(b) If

$$
\delta_{2}=\max \left\{\frac{a_{1}}{a_{0}}, \frac{a_{2}}{a_{1}}\right\}, \delta_{1}=0, \delta_{0}=\max \left\{0, \max _{0 \leq j \leq n-3} \frac{a_{j+3}-\delta_{2} a_{j+2}}{a_{j}}\right\}
$$

or if

$$
\begin{aligned}
& \delta_{2}=\frac{a_{1}}{a_{0}}, \\
& \delta_{1}=\frac{a_{2}-\delta_{2} a_{1}}{a_{0}}, \\
& \delta_{0}=\max \left\{0, \frac{\delta_{1} a_{n}}{-a_{n-1}}, \frac{\delta_{2} a_{n}+\delta_{1} a_{n-1}}{-a_{n-2}}, \max _{0 \leq j \leq n-3} \frac{a_{j+3}-\delta_{2} a_{j+2}-\delta_{1} a_{j+1}}{a_{j}}\right\},
\end{aligned}
$$

then all the zeros of $p$ are excluded from an open disk defined by $|z|<1 / r_{2}$, where $r_{2}$ is the unique positive zero of $z^{3}-\delta_{2} z^{2}-\delta_{1} z-\delta_{0}$.

Proof. Consider $q(z)=\left(z^{3}-\gamma_{2} z^{2}-\gamma_{1} z-\gamma_{0}\right) p(z)$, where $\gamma_{0}, \gamma_{1}, \gamma_{2} \in \mathbb{R}$ :

$$
\begin{aligned}
q(z)=a_{n} z^{n+3}+ & \left(a_{n-1}-\gamma_{2} a_{n}\right) z^{n+2}+\left(a_{n-2}-\gamma_{2} a_{n-1}-\gamma_{1} a_{n}\right) z^{n+1} \\
+ & \sum_{j=3}^{n}\left(a_{j-3}-\gamma_{2} a_{j-2}-\gamma_{1} a_{j-1}-\gamma_{0} a_{j}\right) z^{j}-\left(\gamma_{2} a_{0}+\gamma_{1} a_{1}+\gamma_{0} a_{2}\right) z^{2} \\
& -\left(\gamma_{1} a_{0}+\gamma_{0} a_{1}\right) z-\gamma_{0} a_{0} .
\end{aligned}
$$


If we set

$$
\gamma_{2}=\max \left\{\frac{a_{n-1}}{a_{n}}, \frac{a_{n-2}}{a_{n-1}}\right\}, \gamma_{1}=0, \gamma_{0}=\max \left\{0, \max _{0 \leq j \leq n-3} \frac{a_{j}-\gamma_{2} a_{j+1}}{a_{j+3}}\right\},
$$

or if we set $\gamma_{2}=a_{n-1} / a_{n}, \gamma_{1}=\left(a_{n-2}-\gamma_{2} a_{n-1}\right) / a_{n}$, and

$$
\gamma_{0}=\max \left\{0, \frac{\gamma_{1} a_{0}}{-a_{1}}, \frac{\gamma_{2} a_{0}+\gamma_{1} a_{1}}{-a_{2}}, \max _{0 \leq j \leq n-3} \frac{a_{j}-\gamma_{2} a_{j+1}-\gamma_{1} a_{j+2}}{a_{j+3}}\right\}
$$

then the coefficients of the nonleading powers of $z$ in the right-hand side of (6) are all nonpositive, which means, reasoning as before, that the Cauchy radius of $q$, which is also an upper bound on the moduli of the zeros of $p$, is the unique positive zero of $z^{3}-\gamma_{2} z^{2}-$ $\gamma_{1} z-\gamma_{0}$. This proves part (a).

As before, to prove part (b), we apply the result in part (a) to the reverse polynomial $p^{\#}$, and the proof follows analogously.

Note that, for the first choice of multiplier coefficients in this theorem, $\gamma_{1}$ and $\delta_{1}$ can be negative, and for that first choice we also observe that, if

$$
\frac{a_{n-1}}{a_{n}}=\max _{0 \leq j \leq n-1} \frac{a_{j}}{a_{j+1}} \quad \text { or } \quad \frac{a_{n-2}}{a_{n-1}}=\max _{0 \leq j \leq n-1} \frac{a_{j}}{a_{j+1}}
$$

then $\gamma_{0}=0$ and the upper bound becomes equal to the one from the Eneström-Kakeya theorem. If $a_{n-1} / a_{n} \geq a_{n-2} / a_{n-1}$, this bound will be smaller than the Cauchy radius.

For the second choice of parameters, if

$$
\max _{0 \leq j \leq n-3} \frac{a_{j}}{a_{j+1}} \leq \frac{a_{n-1}}{a_{n}} \leq \frac{a_{n-2}}{a_{n-1}}
$$

then $\gamma_{1} \geq 0$, which implies that $\gamma_{0}=0$, and we obtain precisely the result in Theorem 3.2 , which in this case yields a smaller upper bound than the Cauchy radius, as was explained immediately after the proof of that theorem. Analogous conclusions can be drawn for the lower bound.

Example. We illustrate the theorems in this section with the polynomial $p_{3}(z)=z^{6}+$ $4 z^{5}+2 z^{4}+2 z^{3}+3 z^{2}+6 z+7$. The smallest and largest moduli of its zeros are the endpoints of the interval $[1.075,3.554]$. The different theorems produce the following intervals:

$\begin{array}{ll}\text { Cauchy radii: } & {[0.670,4.580],} \\ \text { Eneström-Kakeya theorem: } & {[0.500,4.000],} \\ \text { Theorem }[3.2 & {[0.633,4.000],} \\ \text { Theorem }[3.3(1): & {[0.766,4.000],} \\ \text { Theorem }[3.3(2): & {[0.807,3.788] .}\end{array}$

The (1) and (2) versions of Theorem 3.3 refer to the first and second choices, respectively, for the parameters in that theorem. Since here $a_{n-1} / a_{n}=\max _{0 \leq j \leq n-1} a_{j} / a_{j+1}$, we find, as predicted, that the Eneström-Kakeya upper bound is the same as that obtained from theorems 3.2 and $3.3(1)$. There is clearly a positive trend in the results when going from the Eneström-Kakeya theorem to Theorem 3.3)(2). 
It is, in general, difficult to predict which of the theorems in this section will produce the best results. Theorems obtained with higher order multipliers do not necessarily outperform those obtained with lower order multipliers, although they frequently do. To gain some insight into their performance, we have carried out a series of numerical comparisons with two classes of randomly generated polynomials of degrees 10 and 40, which allows us to observe the effect of increasing the degree. We have included the Cauchy radii in these comparisons for reference, since they are typically among the best bounds one can expect. The two classes are as follows.

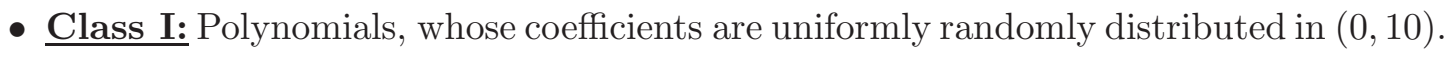

- Class II: Polynomials, whose coefficients are uniformly randomly distributed in $(1,5)$.

The second class of polynomials shows the effect of a more limited range, where the coefficients are bounded away from zero. The results for zero inclusion and exclusion sets (or upper and lower bounds) are very similar and we limit ourselves to the former to avoid overloading the tables below. We generated 1000 polynomials for each class and collected the results in the following two tables.

- Table 1 lists, for Class I polynomials, the median of the ratios of the upper bound to the modulus of the largest zero of the poynomial, i.e., the closer this number is to 1 , the better it is.

- Table 2 is the analog of Table 1 for Class II polynomials.

For Theorem 3.3. the first and second choices of the multiplier are designated in the tables by Theorem 3.3(1) and Theorem 3.3(2), respectively.

\begin{tabular}{c|c|c|c|c|c} 
& Cauchy & Eneström-Kakeya & Theorem 3.2 & Theorem 3.3(1) & Theorem 3.3(2) \\
\hline $\mathrm{n}=10$ & \multirow{2}{*}{1.465} & 4.570 & 1.600 & 1.479 & 1.3553 \\
$\mathrm{n}=40$ & 1.417 & 18.828 & 2.515 & 2.015 & 2.072
\end{tabular}

Table 1: Comparison of upper bounds for Class I polynomials.

\begin{tabular}{c|c|c|c|c|c} 
& Cauchy & Eneström-Kakeya & Theorem 3.2 & Theorem 3.3(1) & Theorem 3.3(2) \\
\hline $\mathrm{n}=10$ & 1.626 & 2.091 & 1.385 & 1.365 & 1.245 \\
$\mathrm{n}=40$ & 1.629 & 2.920 & 1.595 & 1.516 & 1.393
\end{tabular}

Table 2: Comparison of upper bounds for Class II polynomials.

Summarizing these results, we conclude that theorems of the Eneström-Kakeya type perform much better on Class II-like polynomials, where the polynomials have coefficients 
that are more limited in range and that are bounded away from zero. For the latter, this is not surprising, as the expressions for the quantities that define the inclusion regions contain the coefficients in their denominators. For such polynomials these theorems frequently deliver better results than those based on the Cauchy radii. As the degree of the polynomials increases, Cauchy radii tend to become marginally better for Class II polynomials, while they become dramatically better for Class I polynomials.

Furthermore, for both classes of polynomials, Theorems 3.3(1) and 3.3(2) outperform Theorem 3.2. while Theorem 3.3(2) is generally better than Theorem 3.3(1). In all cases, the theorems presented here outperform the Eneström-Kakeya theorem. As a reminder, we note again that the Cauchy radii do not have explicit expressions and need to be computed by solving a polynomial equation.

It is now an entirely straightforward matter to continue the pattern of Theorem 3.2 and Theorem 3.3 with ever higher order multipliers. Of course, beyond a quartic, a numerical method is required to compute their zeros. In fact, such a method may be preferable even in the cubic or quartic case. A simple method such as Newton's method only requires a few iterations for low order polynomials, but whether or not this is practical is, in any event, to be decided elsewhere. In the following section we derive zero inclusion regions that are not centered at the origin.

\section{Single disk not centered at the origin}

So far, we have used polynomial multipliers and the Cauchy radius to derive upper and lower bounds on the moduli of the zeros of a polynomial with positive coefficients. We now derive additional inclusion regions for the zeros of such polynomials, based, this time, on the Cauchy radius of the second kind. The following theorem sets the general tone.

Theorem 4.1. Let the real polynomial $p(z)=\sum_{j=0}^{n} a_{j} z^{j}$ with $n \geq 2$ have positive coefficients, and let

$$
\mu_{1}=\left(\max _{0 \leq j \leq n-2} \frac{a_{j}}{a_{j+2}}\right)^{1 / 2} \text { and } \mu_{2}=\left(\min _{0 \leq j \leq n-2} \frac{a_{j}}{a_{j+2}}\right)^{1 / 2} .
$$

Then all the zeros of $p$ are included in the closed disk

$$
D_{1}=\left\{z \in \mathbb{C}:\left|z+\frac{a_{n-1}}{a_{n}}\right| \leq \frac{a_{n-1}}{a_{n}}+\mu_{1}\right\}
$$

but are excluded from the open disk

$$
D_{2}=\left\{z \in \mathbb{C}:\left|z-\frac{\mu_{2}^{2}}{a_{0} / a_{1}+2 \mu_{2}}\right|<\frac{\mu_{2}\left(a_{0} / a_{1}+\mu_{2}\right)}{a_{0} / a_{1}+2 \mu_{2}}\right\} .
$$

Proof. Consider $q(z)=\left(z^{2}-\gamma\right) p(z)$, where $\gamma \in \mathbb{R}$ :

$$
q(z)=a_{n} z^{n+2}+a_{n-1} z^{n+1}+\sum_{j=2}^{n}\left(a_{j-2}-\gamma a_{j}\right) z^{j}-\gamma a_{1} z-\gamma a_{0} .
$$


Any set containing all the zeros of $q$ will also contain all those of $p$. Equation (7) shows that all the coefficients of $q$, except those of $z^{n+2}$ and $z^{n+1}$, will be nonpositive if

$$
\gamma=\max _{0 \leq j \leq n-2} \frac{a_{j}}{a_{j+2}} .
$$

In that case, the Cauchy radius of the second kind of $q$ is the unique positive solution of $\left(z^{2}-\gamma\right) p(z)=0$, which must be $\sqrt{\gamma}$. The set $\Gamma_{2}(1)$ in Theorem 2.1 then shows that all the zeros of $p$ lie in the disk centered at $-a_{n-1} / a_{n}$ with radius $a_{n-1} / a_{n}+\sqrt{\gamma}$, which is the disk $D_{1}$.

To obtain the zero exclusion disk $D_{2}$, we derive an inclusion disk for the reciprocals of the zeros, which are the zeros of the reverse polynomial $p^{\#}(\zeta)=a_{0} \zeta^{n}+a_{1} \zeta^{n-1}+\cdots+$ $a_{n-1} \zeta+a_{n}$. Proceeding as we did for $D_{1}$, we obtain, by replacing $a_{j}$ by $a_{n-j}$, that the reciprocals of the zeros of $p$ lie in the disk centered at $-a_{1} / a_{0}$ with radius

$$
\left(\max _{0 \leq j \leq n-2} \frac{a_{j+2}}{a_{j}}\right)^{1 / 2}=\left(\min _{0 \leq j \leq n-2} \frac{a_{j}}{a_{j+2}}\right)^{-1 / 2}=\frac{1}{\mu_{2}},
$$

i.e., they lie in the disk

$$
\left\{\zeta \in \mathbb{C}:\left|\zeta+\frac{a_{1}}{a_{0}}\right| \leq \frac{a_{1}}{a_{0}}+\frac{1}{\mu_{2}}\right\} .
$$

With $\zeta=1 / z$, this means that the zeros of $p$ lie in the set

$$
\begin{aligned}
\left\{z \in \mathbb{C}:\left|\frac{1}{z}+\frac{a_{1}}{a_{0}}\right| \leq \frac{a_{1}}{a_{0}}+\frac{1}{\mu_{2}}\right\} & =\left\{z \in \mathbb{C}:\left|1+\frac{a_{1}}{a_{0}} z\right| \leq\left(\frac{a_{1}}{a_{0}}+\frac{1}{\mu_{2}}\right)|z|\right\} \\
& =\left\{z \in \mathbb{C}:\left|z+\frac{a_{0}}{a_{1}}\right| \leq \frac{a_{0}}{a_{1}}\left(\frac{a_{1}}{a_{0}}+\frac{1}{\mu_{2}}\right)|z|\right\} .
\end{aligned}
$$

The inequality defining the set in (8) is of the form $|z+a| \leq|a| R|z|$, where $a=a_{0} / a_{1}$ and $R=a_{1} / a_{0}+1 / \mu_{2}$. Since $R>1 /|a|$, we can apply Lemma 7.1 in the appendix, which shows that the set defined by (8) is the closed exterior of a disk with center $a /\left(|a|^{2} R^{2}-1\right)$ and radius $|a|^{2} R /\left(|a|^{2} R^{2}-1\right)$. This means that the zeros of $p$ are excluded from the open disk with center

$$
\frac{a}{|a|^{2} R^{2}-1}=\frac{a_{0} / a_{1}}{\left(a_{0} / a_{1}\right)^{2}\left(a_{1} / a_{0}+1 / \mu_{2}\right)^{2}-1}=\frac{\mu_{2}^{2}}{a_{0} / a_{1}+2 \mu_{2}}
$$

and, from (9), with radius

$$
\frac{|a|^{2} R}{|a|^{2} R^{2}-1}=\bar{a} R\left(\frac{a}{|a|^{2} R^{2}-1}\right)=\frac{a_{0}}{a_{1}}\left(\frac{a_{1}}{a_{0}}+\frac{1}{\mu_{2}}\right)\left(\frac{\mu_{2}^{2}}{a_{0} / a_{1}+2 \mu_{2}}\right)=\frac{\mu_{2}\left(a_{0} / a_{1}+\mu_{2}\right)}{a_{0} / a_{1}+2 \mu_{2}} .
$$

This completes the proof.

The first part of Theorem 4.1 was also obtained in Corollary 3 of Theorem 3 in [4, although it is formulated slightly differently there with a proof that does not rely on Cauchy-like results, making it more involved algebraically and somewhat difficult to extend beyond the present result. No exclusion disk was included in [4]. 
The sets $D_{1}$ and $D_{2}$ are typical for the kind of results we will obtain in this section, and for subsequent use we define

$$
D(a, \gamma)=\{z \in \mathbb{C}:|z+a| \leq|a|+\gamma\}, \Delta(a, \gamma)=\left\{z \in \mathbb{C}:\left|z-\frac{\gamma^{2}}{|a|+2 \gamma}\right|<\frac{\gamma(|a|+\gamma)}{|a|+2 \gamma}\right\} .
$$

With this notation, $D_{1}$ and $D_{2}$ in the previous theorem are written as $D_{1}=D\left(a_{n-1} / a_{n}, \mu_{1}\right)$ and $D_{2}=\Delta\left(a_{0} / a_{1}, \mu_{2}\right)$.

We now derive two more theorems based on Theorem 2.1. Both serve to illustrate the common thread in their proofs, but each also illustrates a different variation on this theme. The first one shows that the center of the inclusion disk can be varied, while the second theorem shows that even higher order multipliers, if chosen judiciously, can lead to explicitly computable quantities.

In the following theorem, the inclusion region is determined by a disk centered at $-\varepsilon a_{n-1} / a_{n}$, with $0<\varepsilon \leq 1$.

Theorem 4.2. Let the real polynomial $p(z)=\sum_{j=0}^{n} a_{j} z^{j}$ with $n \geq 3$ have positive coefficients, let $0<\varepsilon \leq 1$, and define

$$
\begin{aligned}
\gamma_{2} & =\frac{(1-\varepsilon) a_{n-1}}{a_{n}}, \\
\gamma_{1} & =\frac{a_{n-2}-\gamma_{2} a_{n-1}}{a_{n}}, \\
\gamma_{0} & =\max \left\{0, \frac{\gamma_{1} a_{0}}{-a_{1}}, \frac{\gamma_{2} a_{0}+\gamma_{1} a_{1}}{-a_{2}}, \max _{0 \leq j \leq n-3} \frac{a_{j}-\gamma_{2} a_{j+1}-\gamma_{1} a_{j+2}}{a_{j+3}}\right\},
\end{aligned}
$$

and

$$
\begin{aligned}
& \delta_{2}=\frac{(1-\varepsilon) a_{1}}{a_{0}}, \\
& \delta_{1}=\frac{a_{2}-\delta_{2} a_{1}}{a_{0}} \\
& \delta_{0}=\max \left\{0, \frac{\delta_{1} a_{n}}{-a_{n-1}}, \frac{\delta_{2} a_{n}+\delta_{1} a_{n-1}}{-a_{n-2}}, \max _{0 \leq j \leq n-3} \frac{a_{j+3}-\delta_{2} a_{j+2}-\delta_{1} a_{j+1}}{a_{j}}\right\} .
\end{aligned}
$$

Denote by $\mu_{1}$ the unique positive zero of $z^{3}-\gamma_{2} z^{2}-\gamma_{1} z-\gamma_{0}$ and by $1 / \mu_{2}$ the unique positive zero of $z^{3}-\delta_{2} z^{2}-\delta_{1} z-\delta_{0}$. Then all the zeros of $p$ lie in the closed disk $D\left(\varepsilon a_{n-1} / a_{n}, \mu_{1}\right)$, but are excluded from the open disk $\Delta\left(\frac{1}{\varepsilon} a_{0} / a_{1}, \mu_{2}\right)$.

Proof. Consider $q(z)=\left(z^{3}-\gamma_{2} z^{2}-\gamma_{1} z-\gamma_{0}\right) p(z)$ as in the proof of Theorem 3.3. Then one observes from (6) that, with $\gamma_{0}, \gamma_{1}$, and $\gamma_{2}$ as in the statement of the current theorem, the second-highest coefficient becomes $\varepsilon a_{n-1}>0$, while all the other coefficients are nonpositive. We therefore conclude, as in the proof of Theorem 4.1, that all the zeros of $p$ lie in the closed disk $D\left(\varepsilon a_{n-1} / a_{n}, \mu_{1}\right)$, where $\mu_{1}$ is the unique positive zero of $z^{3}-\gamma_{2} z^{2}-\gamma_{1} z-\gamma_{0}$. The open exclusion disk $\Delta\left(\frac{1}{\varepsilon} a_{0} / a_{1}, \mu_{2}\right)$ follows analogously as before.

When $\varepsilon \rightarrow 0^{+}$, Theorem 4.2 reduces to Theorem 3.3 with the second choice of parameters, and when $\varepsilon=1$, we obtain the following corollary. 
Corollary 4.1. Let the real polynomial $p(z)=\sum_{j=0}^{n} a_{j} z^{j}$ with $n \geq 3$ have positive coefficients, and define

$$
\gamma_{1}=\frac{a_{n-2}}{a_{n}}, \gamma_{0}=\max \left\{0, \max _{0 \leq j \leq n-3} \frac{a_{j}-\gamma_{1} a_{j+2}}{a_{j+3}}\right\}
$$

and

$$
\delta_{1}=\frac{a_{2}}{a_{0}}, \delta_{0}=\max \left\{0, \max _{0 \leq j \leq n-3} \frac{a_{j+3}-\delta_{1} a_{j+1}}{a_{j}}\right\} .
$$

Denote by $\mu_{1}$ the unique positive zero of $z^{3}-\gamma_{1} z-\gamma_{0}$ and by $1 / \mu_{2}$ the unique positive zero of $z^{3}-\delta_{1} z-\delta_{0}$. Then all the zeros of $p$ lie in the closed disk $D\left(a_{n-1} / a_{n}, \mu_{1}\right)$, but are excluded from the open disk $\Delta\left(a_{0} / a_{1}, \mu_{2}\right)$.

Note that $\gamma_{0}=0$ when $a_{n-2} / a_{n}=\max _{0 \leq j \leq n-2} a_{j} / a_{j+2}$, in which case Corollary 4.1 produces the same inclusion disk as in Theorem 4.1. An analogous conclusion follows for the exclusion disk.

The results in the following theorem are obtained by using a quartic multiplier of a particular form that makes it easy to compute its positive zero.

Theorem 4.3. Let the real polynomial $p(z)=\sum_{j=0}^{n} a_{j} z^{j}$ with $n \geq 4$ have positive coefficients, and define

$\alpha=\max \left\{\frac{a_{n-2}}{a_{n}}, \frac{a_{n-3}}{a_{n-1}}\right\}, \beta=\max \left\{0, \max _{0 \leq j \leq n-4} \frac{a_{j}-\alpha a_{j+2}}{a_{j+4}}\right\}, \mu_{1}=\left(\frac{1}{2}\left(\alpha+\left(\alpha^{2}+4 \beta\right)^{1 / 2}\right)\right)^{1 / 2}$,

and

$\gamma=\max \left\{\frac{a_{2}}{a_{0}}, \frac{a_{3}}{a_{1}}\right\}, \delta=\max \left\{0, \max _{0 \leq j \leq n-4} \frac{a_{j+4}-\gamma a_{j+2}}{a_{j}}\right\}, \mu_{2}=\left(\frac{1}{2}\left(\gamma+\left(\gamma^{2}+4 \delta\right)^{1 / 2}\right)\right)^{-1 / 2}$,

Then all the zeros of $p$ lie in the closed disk $D\left(a_{n-1} / a_{n}, \mu_{1}\right)$, but are excluded from the open disk $\Delta\left(a_{0} / a_{1}, \mu_{2}\right)$.

Proof. The proof is similar to that of the previous theorems, except that here we will use a quartic multiplier. Consider $q(z)=\left(z^{4}-\alpha z^{2}-\beta\right) p(z)$ :

$$
\begin{aligned}
q(z)=a_{n} z^{n+4}+a_{n-1} z^{n+3}+ & \left(a_{n-2}-\alpha a_{n}\right) z^{n+2}+\left(a_{n-3}-\alpha a_{n-1}\right) z^{n+1} \\
& +\sum_{j=4}^{n}\left(a_{j-4}-\alpha a_{j-2}-\beta a_{j}\right) z^{j} \\
& \quad-\left(\alpha a_{1}+\beta a_{3}\right) z^{3}-\left(\alpha a_{0}+\beta a_{2}\right) z^{2}-\beta a_{1} z-\beta a_{0} .
\end{aligned}
$$

Reasoning once again as before, we have from equation (10) that all the coefficients of $q$, except those of $z^{n+4}$ and $z^{n+3}$, will be nonpositive if

$$
\alpha=\max \left\{\frac{a_{n-2}}{a_{n}}, \frac{a_{n-3}}{a_{n-1}}\right\} \text { and } \beta=\max \left\{0, \max _{0 \leq j \leq n-4} \frac{a_{j}-\alpha a_{j+2}}{a_{j+4}}\right\} .
$$


Then the Cauchy radius of the second kind of $q$ is the unique positive solution of $\left(z^{4}-\right.$ $\left.\alpha z^{2}-\beta\right) p(z)=0$, which is the unique positive solution $\mu_{1}$ of $z^{4}-\alpha z^{2}-\beta$. This quartic is a quadratic in $z^{2}$, and its positive zero is easily found to be $\mu_{1}=\left(\frac{1}{2}\left(\alpha+\left(\alpha^{2}+4 \beta\right)^{1 / 2}\right)\right)^{1 / 2}$. We then have from Theorem 2.1 with the set $\Gamma_{2}(1)$ that all the zeros of $q$, and therefore also all those of $p$, must lie in the closed disk $D\left(a_{n-1} / a_{n}, \mu_{1}\right)$. As is familiar by now, the open zero exclusion disk $\Delta\left(a_{0} / a_{1}, \mu_{2}\right)$ follows from applying to $p^{\#}$ the result that we just found for $p$.

Here, we observe that, if

$$
\frac{a_{n-2}}{a_{n}}=\max _{0 \leq j \leq n-2} \frac{a_{j}}{a_{j+2}} \quad \text { or } \quad \frac{a_{n-3}}{a_{n-1}}=\max _{0 \leq j \leq n-2} \frac{a_{j}}{a_{j+2}}
$$

then $\beta=0$ and the upper bound becomes $\sqrt{\alpha}$. If, given (11), $a_{n-2} / a_{n} \geq a_{n-3} / a_{n-1}$, then we obtain the same bound as in Theorem 4.1 and Corollary 4.1 while, if, given (11), $a_{n-3} / a_{n-1} \geq a_{n-2} / a_{n}$, then we obtain the same bound as in Theorem 4.1, but not necessarily as in Corollary 4.1. Once again, analogous statements hold for the exclusion disk.

Let us now graphically illustrate some of these results.

Example. Since it would be impractical to form all possible combinations, we have chosen the upper and lower bounds bounds from Theorem 3.3(2) (with the second choice of parameters) and combined them with the inclusion and exclusion disks from Theorem 4.3 for the polynomial $p_{3}(z)=z^{6}+4 z^{5}+2 z^{4}+2 z^{3}+3 z^{2}+6 z+7$ we encountered at the end of Section 3. The result can be found in Figure 1, where the solid circles centered at the origin (right-most circles) are obtained from Theorem 3.3 (2), and the others from Theorem 4.3. The dashed circle indicates the upper bound from the Cauchy radius. The circle obtained from the Cauchy radius of the second kind almost coincides with the one from Theorem 4.3, and is therefore not drawn. The zeros of $p$ are indicated by circled asterisks. Here the disk from Theorem 4.3 cuts off a significant part of the disk from Theorem 3.3 .

The radii of the exclusion and inclusion disks, respectively, for the various theorems used in Figure 1 are as follows:

$\begin{array}{ll}\text { Cauchy radii of the second kind: } & 0.698,5.475, \\ \text { Theorem 4.1; } & 0.513,5.732, \\ \text { Corollary 4.1. } & 0.607,5.618, \\ \text { Theorem 4.3. } & 0.693,5.492\end{array}$

All inclusion disks have the same center $\left(-a_{n-1} / a_{n}\right)$, but the exclusion disks do not. The corresponding radii for the disks from Theorem 4.2, where the inclusion disk is centered at $-\frac{1}{2} a_{n-1} /$ an (obtained with $\varepsilon=1 / 2$ ), are 0.664 and 4.740. As in Section 3, one discerns a positive trend with increasing degree of the multipliers upon which the theorems are based. 


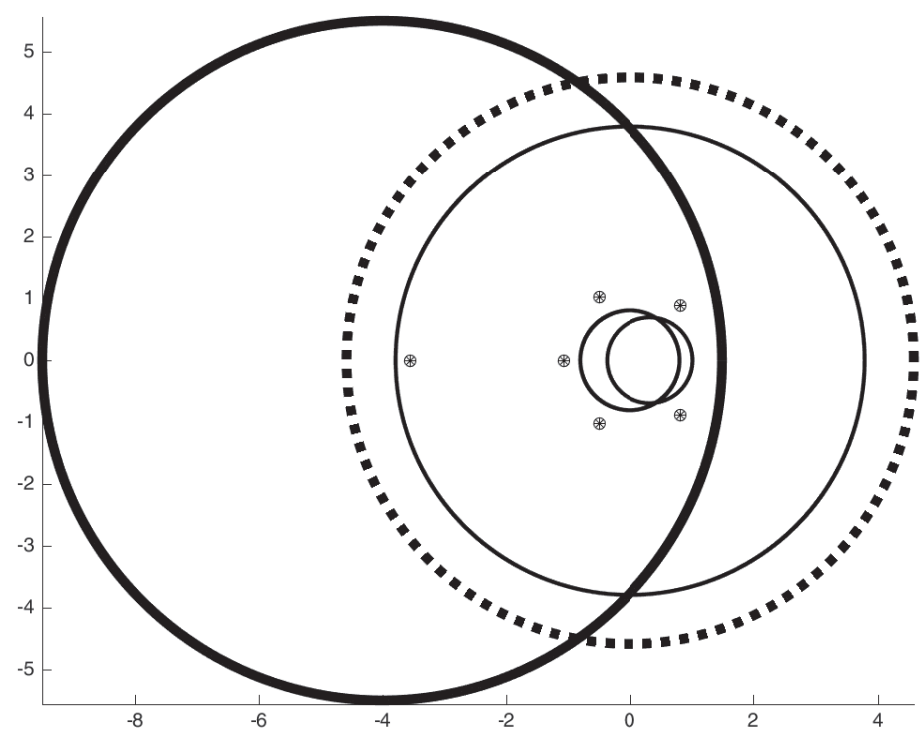

Figure 1: Inclusion regions from Theorems $3.3(2)$ and 4.3 for $p_{3}$.

We now numerically compare the radii of the disks obtained in the theorems of this section using the same two classes of polynomials as in Section 3, Here too, we generated 1000 polynomials for each class and collected the results in the following two tables.

- Table 3 lists, for Class I polynomials, the median of the radii of the disks centered at $-a_{n-1} / a_{n}$.

- Table 4 is the analog of Table 3 for Class II polynomials.

We have designated by "Cauchy" the radius obtained from the set $\Gamma_{2}(1)$ in Theorem 2.1. i.e., $s_{2}+a_{n-1} / a_{n}$, where $s_{2}$ is the Cauchy radius of the second kind.

\begin{tabular}{c|c|c|c|c} 
& Cauchy & Theorem 4.1 & Corollary 4.1 & Theorem 4.3 \\
\hline $\mathrm{n}=10$ & 2.457 & 3.957 & 2.808 & 2.741 \\
$\mathrm{n}=40$ & 2.471 & 7.235 & 3.884 & 3.447
\end{tabular}

Table 3: Comparison of inclusion regions from Section 4 for Class I polynomials. 


\begin{tabular}{c|c|c|c|c} 
& Cauchy & Theorem 4.1 & Corollary 4.1 & Theorem 4.3 \\
\hline $\mathrm{n}=10$ & 2.413 & 2.649 & 2.362 & 2.359 \\
$\mathrm{n}=40$ & 2.403 & 2.857 & 2.463 & 2.411
\end{tabular}

Table 4: Comparison of inclusion regions from Section 4 for Class II polynomials.

We observe that, for both classes of polynomials, Corollary 4.1 and Theorem 4.3 outperform Theorem 4.1. Corollary 4.1 is better than Theorem 4.3 for Class I polynomials, but the reverse is true for Class II polynomials with increasing degree of the polynomials. The disk based on the Cauchy radius of the second kind (which requires the solution of a polynomial equation) is generally smaller than any of the disks obtained here, especially when the degree of the polynomial increases.

So far, we have only used the sets $\Gamma_{1}(k)$ and $\Gamma_{2}(k)$ from Theorem 2.1 for $k=1$. In the following two sections we derive sets for $k \geq 2$ consisting of two and three disks, respectively. We limit ourselves to zero inclusion regions obtained using the polynomial, but not the reverse polynomial.

\section{Two disks}

In this section we derive zero inclusion regions consisting of two disks, organized in three theorems. The first of these relies on a quadratic multiplier, while the other two relie on cubic multipliers.

Theorem 5.1. Let the real polynomial $p(z)=\sum_{j=0}^{n} a_{j} z^{j}$ with $n \geq 2$ have positive coefficients, and define

$$
\mu=\left(\max _{0 \leq j \leq n-2} \frac{a_{j}}{a_{j+2}}\right)^{1 / 2} \text { and } R=\mu^{2}+\frac{a_{n-1}}{a_{n}} \mu .
$$

Then all the zeros of $p$ are included in the union of disks

$$
\left\{z \in \mathbb{C}:|z| \leq R^{1 / 2}\right\} \bigcup\left\{z \in \mathbb{C}:\left|z+\frac{a_{n-1}}{a_{n}}\right| \leq R^{1 / 2}\right\}
$$

If the disks are disjoint, then the disk centered at $-a_{n-1} / a_{n}$ contains one zero and the one centered at the origin contains the remaining $n-1$ zeros of $p$.

Proof. Consider, as in the proof of Theorem 4.1, the polynomial $q(z)=\left(z^{2}-\gamma\right) p(z)$. Then

$$
q(z)=a_{n} z^{n+2}+a_{n-1} z^{n+1}+\sum_{j=2}^{n}\left(a_{j-2}-\gamma a_{j}\right) z^{j}-\gamma a_{1} z-\gamma a_{0} .
$$

If we now choose $\gamma=\mu^{2}$ with $\mu$ as in the statement of the theorem, then all the coefficients of $q$, other than the two leading ones, are nonpositive, so that $s_{2}$, its Cauchy radius of the 
second kind is its unique positive solution, namely, $\mu$. The set $\Gamma_{1}(2)$ in Theorem 2.1 is then given by

$$
\Gamma_{1}(2)=\left\{z \in \mathbb{C}:\left|z\left(z+\frac{a_{n-1}}{a_{n}}\right)\right| \leq R\right\}
$$

with $R=\mu^{2}+\left(a_{n-1} / a_{n}\right) \mu$. This set is contained in the following union of two disks:

$$
\left\{z \in \mathbb{C}:|z| \leq R^{1 / 2}\right\} \bigcup\left\{z \in \mathbb{C}:\left|z+\frac{a_{n-1}}{a_{n}}\right| \leq R^{1 / 2}\right\}
$$

If the disks are disjoint, then from Theorem 2.1 with $k=2$, we obtain that the disk centered at the origin contains $1+(n+2)-2=n+1$ zeros, while the disk centered at $-a_{n-1} / a_{n}$ contains one zero of $q$. The zeros of $q$ are those of $p$ with the addition of $\pm \mu$. Because the disks are disjoint, all points in the disk centered at $-a_{n-1} / a_{n}$ have a negative real part, so that $\mu$ must lie in the disk centered at the origin, which means that $-\mu$ also lies in that disk. The only zero of $q$ in the disk centered at $-a_{n-1} / a_{n}$ must therefore be a zero of $p$, while the other $n-1$ zeros of $p$ lie in the disk centered at the origin. This concludes the proof.

This theorem is closely related to Theorem 4.1. However, instead of one disk centered at $-a_{n-1} / a_{n}$, we now have two disks - one centered at the origin and the other at $-a_{n-1} / a_{n}$ - with a smaller radius. That this radius is smaller follows from the fact that

$$
\mu^{2}+\frac{a_{n-1}}{a_{n}} \mu \leq\left(\mu+\frac{a_{n-1}}{a_{n}}\right)^{2} \Longrightarrow\left(\mu^{2}+\frac{a_{n-1}}{a_{n}} \mu\right)^{1 / 2} \leq \mu+\frac{a_{n-1}}{a_{n}}
$$

where $\mu$ has the same meaning here as in Theorem 4.1.

Theorem 5.2. Let the real polynomial $p(z)=\sum_{j=0}^{n} a_{j} z^{j}$ with $n \geq 3$ have positive coefficients, let $0<\varepsilon \leq 1$, and define

$$
\gamma_{2}=\frac{(1-\varepsilon) a_{n-1}}{a_{n}}, \gamma_{1}=\frac{a_{n-2}-\gamma_{2} a_{n-1}}{a_{n}}
$$

and

$$
\gamma_{0}=\max \left\{0, \frac{\gamma_{1} a_{0}}{-a_{1}}, \frac{\gamma_{2} a_{0}+\gamma_{1} a_{1}}{-a_{2}}, \max _{0 \leq j \leq n-3} \frac{a_{j}-\gamma_{2} a_{j+1}-\gamma_{1} a_{j+2}}{a_{j+3}}\right\}
$$

Let $\mu$ be the unique positive zero of $z^{3}-\gamma_{2} z^{2}-\gamma_{1} z-\gamma_{0}$, and let $R=\mu^{3}+\left(\varepsilon a_{n-1} / a_{n}\right) \mu^{2}$. Then all the zeros of $p$ are included in the union of disks

$$
\left\{z \in \mathbb{C}:|z| \leq R^{1 / 3}\right\} \bigcup\left\{z \in \mathbb{C}:\left|z+\varepsilon \frac{a_{n-1}}{a_{n}}\right| \leq R^{1 / 3}\right\}
$$

If the disks are disjoint, then the disk centered at $-\varepsilon a_{n-1} / a_{n}$ contains one zero and the one centered at the origin contains the remaining $n-1$ zeros of $p$. 
Proof. As in the proof of Theorems 3.3 and 4.2, consider the polynomial $q(z)=$ $\left(z^{3}-\gamma_{2} z^{2}-\gamma_{1} z-\gamma_{0}\right) p(z)$ :

$$
\begin{array}{r}
q(z)=a_{n} z^{n+3}+\left(a_{n-1}-\gamma_{2} a_{n}\right) z^{n+2}+\left(a_{n-2}-\gamma_{2} a_{n-1}-\gamma_{1} a_{n}\right) z^{n+1} \\
+\sum_{j=3}^{n}\left(a_{j-3}-\gamma_{2} a_{j-2}-\gamma_{1} a_{j-1}-\gamma_{0} a_{j}\right) z^{j}-\left(\gamma_{2} a_{0}+\gamma_{1} a_{1}+\gamma_{0} a_{2}\right) z^{2} \\
-\left(\gamma_{1} a_{0}+\gamma_{0} a_{1}\right) z-\gamma_{0} a_{0} .
\end{array}
$$

If we choose $\gamma_{0}, \gamma_{1}$, and $\gamma_{2}$ as in the statement of the theorem, then the two leading coefficents are positive, the coefficient of $z^{n+1}$ vanishes and all other coefficients are nonpositive. The Cauchy radius of the third kind of $q$ is its unique positive zero, which is the unique positive zero $\mu$ of $z^{3}-\gamma_{2} z^{2}-\gamma_{1} z-\gamma_{0}$. This means that the set $\Gamma_{1}(3)$ in Theorem 2.1 is given by

$$
\Gamma_{1}(3)=\left\{z \in \mathbb{C}:\left|z^{2}\left(z+\varepsilon \frac{a_{n-1}}{a_{n}}\right)\right| \leq R\right\},
$$

where $R=\mu^{3}+\left(a_{n-1} / a_{n}\right) \mu^{2}$. This set is contained in the following union of two disks:

$$
\left\{z \in \mathbb{C}:|z| \leq R^{1 / 3}\right\} \bigcup\left\{z \in \mathbb{C}:\left|z+\varepsilon \frac{a_{n-1}}{a_{n}}\right| \leq R^{1 / 3}\right\}
$$

If the disks are disjoint, then from Theorem 2.1 with $k=3$, we have that the disk centered at the origin contains $2+(n+3)-3=n+2$ zeros, while the disk centered at $-\varepsilon a_{n-1} / a_{n}$ contains one zero of $q$. The zeros of $q$ are those of $p$ with the addition of the three zeros of $h(z)=z^{3}-\gamma_{2} z^{2}-\gamma_{1} z-\gamma_{0}$. This polynomial $h$ has a unique positive zero, which we denoted by $\mu$, and its coefficients $\gamma_{2}$ and $\gamma_{0}$ are nonnegative. Let us first assume that $\gamma_{1}$ is also nonnegative. Then $\mu$ has the largest modulus of all the zeros of $h$. Because the disks are disjoint, all points in the disk centered at $-\varepsilon a_{n-1} / a_{n}$ have a negative real part, so that $\mu$ must lie in the disk centered at the origin. Since it has the largest modulus of all zeros of $h$, the other two zeros must also lie in that disk, so that the only zero of $q$ in the disk centered at $-\varepsilon a_{n-1} / a_{n}$ must be a zero of $p$, while the other $n-1$ zeros of $p$ lie in the disk centered at the origin. of

If $\gamma_{1}<0$, then $h$ has no negative zeros, since these negative zeros are the positive zeros

$$
(-\zeta)^{3}-\gamma_{2}(-\zeta)^{2}-\gamma_{1}(-\zeta)-\gamma_{0}=-\zeta^{3}-\gamma_{2} \zeta^{2}+\gamma_{1} \zeta-\gamma_{0}
$$

and this is a polynomial with nonpositive coefficients, which does not have positive zeros. The other two zeros of $h$ must therefore be complex (and each other's conjugate). If we denote these complex zeros by $\nu$ and $\bar{\nu}$, then we have that

$$
\mu+\nu+\bar{\nu}=\gamma_{2} \Longrightarrow \mu+2 \operatorname{Re}(\nu)>0 .
$$

If $\operatorname{Re}(\nu) \geq 0$, then $\nu$ and $\bar{\nu}$ cannot lie in the disk centered at $-\varepsilon a_{n-1} / a_{n}$, and must therefore be in the disk centered at the origin. If $\operatorname{Re}(\nu)<0$, then from (12), we have that $\operatorname{Re}(\nu)>-\mu / 2$, which means that $\nu$ and $\bar{\nu}$ must lie in the disk centered at the origin 
because that disk contains $\mu$. We conclude that the only zero of $q$ in the disk centered at $-\varepsilon a_{n-1} / a_{n}$ must be a zero of $p$. The remaining $n-1$ zeros of $p$ are contained in the disk centered at the origin. This concludes the proof.

When $\varepsilon \rightarrow 0^{+}$, Theorem 5.2 reduces to Theorem 3.3 with the second choice of parameters, and when $\varepsilon=1$, we obtain the following corollary.

Corollary 5.1. Let the real polynomial $p(z)=\sum_{j=0}^{n} a_{j} z^{j}$ with $n \geq 3$ have positive coefficients. Define

$$
\gamma_{1}=\frac{a_{n-2}}{a_{n}} \text { and } \gamma_{0}=\max \left\{0, \max _{0 \leq j \leq n-3} \frac{a_{j}-\gamma_{1} a_{j+2}}{a_{j+3}}\right\}
$$

Let $\mu$ be the unique positive zero of $z^{3}-\gamma_{1} z-\gamma_{0}$, and let $R=\mu^{3}+\left(a_{n-1} / a_{n}\right) \mu^{2}$.

Then all the zeros of $p$ are included in the union of disks

$$
\left\{z \in \mathbb{C}:|z| \leq R^{1 / 3}\right\} \bigcup\left\{z \in \mathbb{C}:\left|z+\frac{a_{n-1}}{a_{n}}\right| \leq R^{1 / 3}\right\}
$$

If the disks are disjoint, then the disk centered at $-a_{n-1} / a_{n}$ contains one zero and the one centered at the origin contains the remaining $n-1$ zeros of $p$.

Remarks. Theorem 5.2 is similar to Theorem 4.2. However, we now have two disks instead of one, both with the same radius, and this radius is smaller than that of the disk in Theorem 4.2 because

$$
\mu^{3}+\varepsilon \frac{a_{n-1}}{a_{n}} \mu^{2} \leq\left(\mu+\varepsilon \frac{a_{n-1}}{a_{n}}\right)^{3} \Longrightarrow\left(\mu^{3}+\varepsilon \frac{a_{n-1}}{a_{n}} \mu^{2}\right)^{1 / 3} \leq \mu+\varepsilon \frac{a_{n-1}}{a_{n}} .
$$

A similar observation holds for Corollary 5.1 and Corollary 4.1.

Theorem 5.3. Let the real polynomial $p(z)=\sum_{j=0}^{n} a_{j} z^{j}$ with $n \geq 3$ have positive coefficients, and define

$$
\mu=\left(\max _{0 \leq j \leq n-3} \frac{a_{j}}{a_{j+3}}\right)^{1 / 3} \text { and } R=\mu^{2}+\frac{a_{n-1}}{a_{n}} \mu+\frac{a_{n-2}}{a_{n}},
$$

and let $c_{1}$ and $c_{2}$ be the zeros of the quadratic $z^{2}+\left(a_{n-1} / a_{n}\right) z+a_{n-2} / a_{n}$. Then all the zeros of $p$ are included in the union of disks

$$
\left\{z \in \mathbb{C}:\left|z-c_{1}\right| \leq R^{1 / 2}\right\} \bigcup\left\{z \in \mathbb{C}:\left|z-c_{2}\right| \leq R^{1 / 2}\right\}
$$

If the disks are disjoint, then $c_{2}<c_{1}<0$, the disk centered at $c_{2}$ contains one zero, and the one centered at $c_{1}$ contains the remaining $n-1$ zeros of $p$.

Proof. Let the polynomial $q$ be defined by $q(z)=\left(z^{3}-\gamma\right) p(z)$. Then

$$
q(z)=a_{n} z^{n+3}+a_{n-1} z^{n+2}+a_{n-2} z^{n+1}+\sum_{j=3}^{n}\left(a_{j-3}-\gamma a_{j}\right) z^{j}-\gamma a_{2} z^{2}-\gamma a_{1} z-\gamma a_{0} .
$$


If $\gamma=\mu^{3}$ with $\mu$ as in the statement of the theorem, then all the coefficients of $q$, other than the three leading ones, are nonpositive, so that its Cauchy radius of the third kind is its unique positive zero, namely, $\mu$. The set $\Gamma_{2}(2)$ in Theorem 2.1 is then given by

$$
\Gamma_{2}(2)=\left\{z \in \mathbb{C}:\left|z^{2}+\frac{a_{n-1}}{a_{n}} z+\frac{a_{n-2}}{a_{n}}\right| \leq R\right\}
$$

with $R=\mu^{2}+\left(a_{n-1} / a_{n}\right) \mu+a_{n-2} / a_{n}$. Let

$$
c_{1}=\frac{1}{2}\left(-\frac{a_{n-1}}{a_{n}}+\left(\frac{a_{n-1}^{2}}{a_{n}^{2}}-4 \frac{a_{n-2}}{a_{n}}\right)^{1 / 2}\right) \text { and } c_{2}=\frac{1}{2}\left(-\frac{a_{n-1}}{a_{n}}-\left(\frac{a_{n-1}^{2}}{a_{n}^{2}}-4 \frac{a_{n-2}}{a_{n}}\right)^{1 / 2}\right)
$$

be the zeros of $z^{2}+\left(a_{n-1} / a_{n}\right) z+a_{n-2} / a_{n}$, then this set is contained in the following union of two disks:

$$
\left\{z \in \mathbb{C}:\left|z-c_{1}\right| \leq R^{1 / 2}\right\} \bigcup\left\{z \in \mathbb{C}:\left|z-c_{2}\right| \leq R^{1 / 2}\right\}
$$

These disks are disjoint if and only if

$$
\left|c_{1}-c_{2}\right|=\left|\left(\frac{a_{n-1}^{2}}{a_{n}^{2}}-4 \frac{a_{n-2}}{a_{n}}\right)^{1 / 2}\right|>2 R^{1 / 2}
$$

If $a_{n-1}^{2} / a_{n}^{2} \leq 4 a_{n-2} / a_{n}$, then

$$
\left|c_{1}-c_{2}\right|=\left|\left(\frac{a_{n-1}^{2}}{a_{n}^{2}}-4 \frac{a_{n-2}}{a_{n}}\right)^{1 / 2}\right|=\left|\left(4 \frac{a_{n-2}}{a_{n}}-\frac{a_{n-1}^{2}}{a_{n}^{2}}\right)^{1 / 2} i\right|=\left(4 \frac{a_{n-2}}{a_{n}}-\frac{a_{n-1}^{2}}{a_{n}^{2}}\right)^{1 / 2} .
$$

In this case, it is impossible that $\left|c_{1}-c_{2}\right|>2 R^{1 / 2}$ since $\left|c_{1}-c_{2}\right|<2\left(a_{n-2} / a_{n}\right)^{1 / 2}$, while $2 R^{1 / 2}>2\left(a_{n-2} / a_{n}\right)^{1 / 2}$. This means that when the disks are disjoint, then $a_{n-1}^{2} / a_{n}^{2}>$ $4 a_{n-2} / a_{n}$ and both $c_{1}$ and $c_{2}$ are real and negative with $c_{2}<c_{1}<0$.

The disk centered at $c_{1}$ must contain the origin since otherwise, by Theorem 2.1, the set $\Gamma_{2}(2)$ would only contain two zeros of $q$, when it has, in fact, $n+3$ zeros. This can also be shown explicitly. As a consequence, the disk centered at $c_{1}$ contains $1+(n+3)-2=n+2$ zeros, while the disk centered at $c_{2}$ contains one zero of $q$. Since $\mu>0$, it must lie in the disk centered at $c_{1}$ and because the other two zeros of $z^{3}-\mu^{3}$ both have the same modulus $\mu$, they too must lie in this disk. Therefore, the single zero of $q$ that lies in the disk centered at $c_{2}$ cannot be a zero of $z^{3}-\mu^{3}$ and must therefore be a zero of $p$. Clearly, the remaining $n-1$ zeros of $p$ lie in the disk centered at $c_{1}$. This concludes the proof.

It is generally difficult to predict which theorem produces the better result, but to obtain some idea, we numerically compare the radii of the disks from Theorem 5.1 and Corollary 5.1, as they have the same centers. We generated 1000 polynomials for each of the same two classes as in Section 3, and listed the results in the following two tables.

- Table 5 lists, for Class I polynomials, the median of the radii of the disks centered at the origin and $-a_{n-1} / a_{n}$. 
- Table 6 is the analog of Table 5 for Class II polynomials.

We have designated by "Cauchy" the radius obtained from the set $\Gamma_{1}(2)$ in Theorem 2.1 , i.e., $\left(s_{2}^{2}+\left(a_{n-1} / a_{n}\right) s_{2}\right)^{1 / 2}$, where $s_{2}$ is the Cauchy radius of the second kind.

\begin{tabular}{c|c|c|c|} 
& Cauchy & Theorem 5.1 & Corollary 5.1 \\
\hline $\mathrm{n}=10$ & 1.845 & 3.315 & 2.052 \\
$\mathrm{n}=40$ & 1.846 & 6.292 & 2.884
\end{tabular}

Table 5: Comparison of inclusion regions from Section 5 for Class I polynomials.

\begin{tabular}{c|c|c|c|} 
& Cauchy & Theorem 5.1 & Corollary 5.1 \\
\hline $\mathrm{n}=10$ & 1.845 & 2.072 & 1.633 \\
$\mathrm{n}=40$ & 1.854 & 2.360 & 1.795
\end{tabular}

Table 6: Comparison of inclusion regions from Section 5 for Class II polynomials.

We observe that, for both classes of polynomials, Corollary 5.1 generally outperforms Theorem 5.1, although on rare occasions the opposite is true. The disks based on the Cauchy radius of the second kind, which, we recall, requires the solution of a polynomial equation, are generally smaller than any of the disks obtained here for Class I polynomials, but not for Class II polynomials, where Corollary 5.1 clearly delivers better results on average.

Example. Figure 2 compares the inclusion regions obtained from Theorem 4.1 and Theorem 5.1 for the same polynomial $p_{3}(z)=z^{6}+4 z^{5}+2 z^{4}+2 z^{3}+3 z^{2}+6 z+7$ that we used at the end of Section 3 and in Figure 1. The dotted circle centered at the origin (with radius 3.788) is the boundary of the disk obtained from Theorem 3.3 (2), while the large dashed circle (with radius 5.732) is the boundary of the disk from Theorem 4.1. The two smaller solid circles (with radii 3.151) are the boundaries of the disks from Theorem 5.1 . The zeros of $p$ are indicated by circled asterisks. The Cauchy radius of $p_{3}$ is 4.580 . 


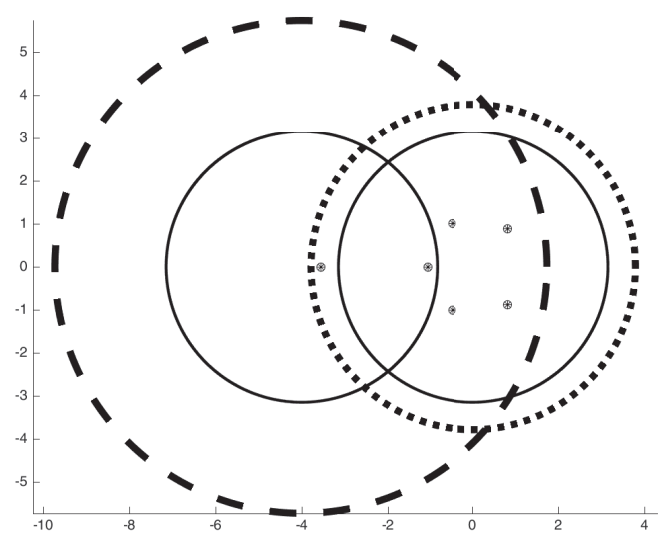

Figure 2: Comparison of Theorems 4.1 and 5.1 for $p_{3}$.

Regions composed of two disks are not necessarily smaller than those composed of a single disk, although they frequently are. Moreover, when the disks are disjoint, they provide additional information about the location of the zeros that cannot be obtained from standard generalizations of the Eneström-Kakeya theorem.

\section{Three disks}

In this section we carry out one more application of Theorem 2.1 to obtain zero inclusion regions consisting of three disks.

Theorem 6.1. Let the real polynomial $p(z)=\sum_{j=0}^{n} a_{j} z^{j}$ with $n \geq 3$ have positive coefficients. Let $0<\varepsilon \leq 1$, and define

$$
\gamma_{1}=\frac{(1-\varepsilon) a_{n-2}}{a_{n}} \text { and } \gamma_{0}=\max \left\{0, \max _{0 \leq j \leq n-3} \frac{a_{j}-\gamma_{1} a_{j+2}}{a_{j+3}}\right\}
$$

Let $\mu$ be the unique positive zero of $z^{3}-\gamma_{1} z-\gamma_{0}$, let $R=\mu^{3}+\left(a_{n-1} / a_{n}\right) \mu^{2}+\varepsilon\left(a_{n-2} / a_{n}\right) \mu$, and let $c_{1}(\varepsilon)$ and $c_{2}(\varepsilon)$ be the zeros of the quadratic $z^{2}+\left(a_{n-1} / a_{n}\right) z+\varepsilon a_{n-2} / a_{n}$.

Then all the zeros of $p$ are included in the union of disks

$$
\left\{z \in \mathbb{C}:|z| \leq R^{1 / 3}\right\} \bigcup\left\{z \in \mathbb{C}:\left|z-c_{1}(\varepsilon)\right| \leq R^{1 / 3}\right\} \bigcup\left\{z \in \mathbb{C}:\left|z-c_{2}(\varepsilon)\right| \leq R^{1 / 3}\right\} .
$$

There exist only the following two scenarios for disks to be disjoint.

(1) The disk centered at the origin is disjoint from the other two, in which case that disk contains $n-2$ zeros of $p$, while the union of the other two contains the two remaining zeros of $p$. If these are also disjoint, then each contains one zero of $p$. 
(2) The two disks not centered at the orgin are disjoint, but only one of them is disjoint from the disk at the origin, in which case that disk contains one zero of $p$, while the union of the other two contains $n-1$ zeros of $p$. This scenario is only possible when $c_{1}(\varepsilon)$ and $c_{2}(\varepsilon)$ are real and negative.

Proof. Consider the polynomial $q(z)=z^{3}-\gamma_{1} z-\gamma_{0}$, given by

$$
\begin{aligned}
q(z)=a_{n} z^{n+3}+a_{n-1} z^{n+2}+\left(a_{n-2}-\gamma_{1} a_{n}\right) z^{n+1} & \\
+\sum_{j=3}^{n}\left(a_{j-3}-\gamma_{1} a_{j-1}-\gamma_{0} a_{j}\right) z^{j}-\left(\gamma_{1} a_{1}+\gamma_{0} a_{2}\right) z^{2} & -\left(\gamma_{1} a_{0}+\gamma_{0} a_{1}\right) z-\gamma_{0} a_{0} .
\end{aligned}
$$

If we choose $\gamma_{0}$ and $\gamma_{1}$ as in the statement of the theorem, then the three leading coefficents are positive, while all other coefficients are nonpositive. The Cauchy radius of the third kind of $q$ is then its unique positive zero, which is the unique positive zero $\mu$ of $z^{3}-\gamma_{1} z-\gamma_{0}$. This means that the set $\Gamma_{1}(3)$ in Theorem 2.1 is given by

$$
\Gamma_{1}(3)=\left\{z \in \mathbb{C}:\left|z\left(z^{2}+\frac{a_{n-1}}{a_{n}} z+\varepsilon \frac{a_{n-2}}{a_{n}}\right)\right| \leq R\right\}
$$

where $R=\mu^{3}+\left(a_{n-1} / a_{n}\right) \mu^{2}+\varepsilon\left(a_{n-2} / a_{n}\right) \mu$. This set is contained in the following union of three disks:

$$
\left\{z \in \mathbb{C}:|z| \leq R^{1 / 3}\right\} \bigcup\left\{z \in \mathbb{C}:\left|z-c_{1}(\varepsilon)\right| \leq R^{1 / 3}\right\} \bigcup\left\{z \in \mathbb{C}:\left|z-c_{2}(\varepsilon)\right| \leq R^{1 / 3}\right\} .
$$

Several scenarios arise when the disks are disjoint. If

$$
\frac{a_{n-1}^{2}}{a_{n}^{2}} \leq 4 \frac{a_{n-2}}{a_{n}}
$$

then $c_{1}(\varepsilon)$ and $c_{1}(\varepsilon)$ are complex conjugate with a negative real part, and the disks centered at these points are either not disjoint or both disjoint from the disk centered at the origin. When they are disjoint, then $q$ has $1+(n+3)-3=n+1$ zeros in the disk centered at the orgin. One of those must be $\mu$, which is the real positive zero of $h(z):=z^{3}-\gamma_{1} z-\gamma_{0}$ with largest modulus. The other two zeros of $h$ must therefore also lie in that disk, and this means that the remaining $n-2$ zeros of $q$ in that disk are zeros of $p$, while two zeros of $p$ lie in the union of the two disks centered at $c_{1}(\varepsilon)$ and $c_{2}(\varepsilon)$. If these are also disjoint from each other, then each contains one zero of $p$.

If $c_{1}(\varepsilon)$ and $c_{1}(\varepsilon)$ are not complex, then they are both real and negative. If the disks centered at these points are both disjoint from the disk centered at the origin, then reasoning similarly as before, their union contains two zeros of $p$; if they are disjoint from each other, then each contains one zero of $p$. If only one is disjoint from the disk centered at the origin, then it contains one zero of $p$.

When $\varepsilon \rightarrow 0^{+}$, Theorem 6.1 reduces to Corollary [5.1, and when $\varepsilon=1$, we obtain the following corollary. 
Corollary 6.1. Let the real polynomial $p(z)=\sum_{j=0}^{n} a_{j} z^{j}$ with $n \geq 3$ have positive coefficients. Define

$$
\mu=\left(\max _{0 \leq j \leq n-3} \frac{a_{j}}{a_{j+3}}\right)^{1 / 3} \text { and } R=\mu^{3}+\frac{a_{n-1}}{a_{n}} \mu^{2}+\frac{a_{n-2}}{a_{n}} \mu,
$$

and let $c_{1}$ and $c_{2}$ be the zeros of the quadratic $z^{2}+\left(a_{n-1} / a_{n}\right) z+a_{n-2} / a_{n}$.

Then all the zeros of $p$ are included in the union of disks

$$
\left\{z \in \mathbb{C}:|z| \leq R^{1 / 3}\right\} \bigcup\left\{z \in \mathbb{C}:\left|z-c_{1}\right| \leq R^{1 / 3}\right\} \bigcup\left\{z \in \mathbb{C}:\left|z-c_{2}\right| \leq R^{1 / 3}\right\} .
$$

There exist only the following two scenarios for disks to be disjoint.

(1) The disk centered at the origin is disjoint from the other two, in which case that disk contains $n-2$ zeros of $p$, while the union of the other two contains the two remaining zeros of $p$. If these are also disjoint, then each contains one zero of $p$.

(2) The two disks not centered at the orgin are disjoint, but only one of them is disjoint from the disk at the origin, in which case that disk contains one zero of $p$, while the union of the other two contains $n-1$ zeros of $p$. This scenario is only possible when $c_{1}$ and $c_{2}$ are real and negative.

\section{Remarks.}

- We remark that the two disks in Corollary 6.1 that are not centered at the origin have the same centers as the disks in Theorem 5.3 , but their radii are smaller. This is so because

$$
\mu^{2}\left(\mu^{2}+\frac{a_{n-1}}{a_{n}} \mu+\frac{a_{n-2}}{a_{n}}\right)^{2} \leq\left(\mu^{2}+\frac{a_{n-1}}{a_{n}} \mu+\frac{a_{n-2}}{a_{n}}\right)^{3}
$$

implies that

$$
\mu^{1 / 3}\left(\mu^{2}+\frac{a_{n-1}}{a_{n}} \mu+\frac{a_{n-2}}{a_{n}}\right)^{1 / 3} \leq\left(\mu^{2}+\frac{a_{n-1}}{a_{n}} \mu+\frac{a_{n-2}}{a_{n}}\right)^{1 / 2} .
$$

- If the radius of the disk centered at the origin in the theorems both in this and the previous section is larger than the Cauchy radius, then it will obviously contain all the zeros of the polynomial and the other $\operatorname{disk}(\mathrm{s})$ can be ignored. This can easily be detected by a simple substitution of the radius of that disk in equation (10).

\section{Examples.}

- In Figure 3, we compare the inclusion regions obtained from Theorem 5.1 and Theorem 6.1 for the same polynomial $p_{3}(z)=z^{6}+4 z^{5}+2 z^{4}+2 z^{3}+3 z^{2}+6 z+7$ we used before. The dotted circle centered at the origin (with radius 3.788 ) is the boundary of the disk obtained from Theorem 3.3(2). The solid circles in the figure on the left (with radii 3.151) are those obtained from Theorem 5.1, while those in the figure on the right (with radii 2.507) are obtained from Theorem 6.1. The zeros of $p$ are indicated by circled asterisks. The Cauchy radius in this case is 4.580 . 
- In Figure 4, we carried out the same comparison as in Figure 3 for the polynomial $p_{4}(z)=z^{6}+9 z^{5}+5 z^{4}+6 z^{3}+4 z^{2}+8 z+1$. The dotted circle centered at the origin (with radius 8.744) is the boundary of the disk obtained from Theorem 3.3)(2). The solid circles in the figure on the left (with radii 5.012) are those obtained from Theorem 5.1, while those in the figure on the right (with radii 3.552) are obtained from Theorem 6.1. As before, the zeros of $p$ are indicated by circled asterisks. The Cauchy radius of $p_{4}$ is 9.592. Here Theorem 6.1 isolates one zero of $p_{4}$ from the others.
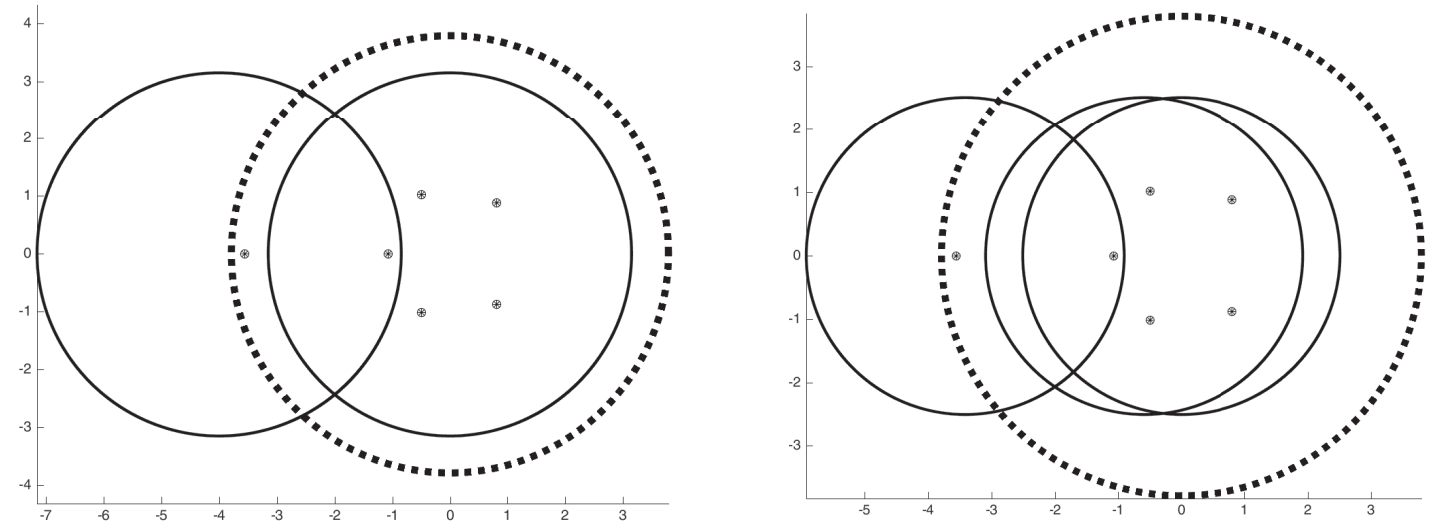

Figure 3: Comparison of Theorems 5.1 and 6.1 for $p_{3}$.


Figure 4: Comparison of Theorems 5.1 and 6.1 for $p_{4}$.

Theorem 6.1 is just one more example of the kind of results that can be generated in the framework we have established, which allows for many variations. More disks can be obtained by combining various multipliers with Theorem 2.1. 
Conclusion. We have constructed a framework to derive generalizations of the classical Eneström-Kakeya theorem using two simple tools: polynomial multipliers and a theorem establishing inclusion regions for the zeros of a polynomial. This framework unifies and simplifies the derivation of these generalizations, obtaining new as well as old results in the process, while transparently showing how more such results can be generated. One feature of our results, namely, zero inclusion regions consisting of more than one disk, is not found in any of the existing generalizations of this theorem.

\section{Appendix}

We state and prove a lemma that was used in Section 4

Lemma 7.1. The set $\{z \in \mathbb{C}:|z+a| \leq|a| R|z|\}$, where $a \in \mathbb{C}$ and $R>1 /|a|$, is the closed exterior of a disk with center $a /\left(|a|^{2} R^{2}-1\right)$ and radius $|a|^{2} R /\left(|a|^{2} R^{2}-1\right)$.

Proof.

$$
\begin{aligned}
|z+a| \leq|a| R|z| & \Longleftrightarrow|z+a|^{2} \leq|a|^{2} R^{2}|z|^{2} \\
& \Longleftrightarrow|z|^{2}+2 \operatorname{Re}(\bar{a} z)+|a|^{2} \leq|a|^{2} R^{2}|z|^{2} \\
& \Longleftrightarrow 0 \leq\left(|a|^{2} R^{2}-1\right)|z|^{2}-2 R e(\bar{a} z)-|a|^{2} \\
& \Longleftrightarrow 0 \leq|z|^{2}-\frac{2 R e(\bar{a} z)}{|a|^{2} R^{2}-1}-\frac{|a|^{2}}{|a|^{2} R^{2}-1} \\
& \Longleftrightarrow 0 \leq\left|z-\frac{a}{|a|^{2} R^{2}-1}\right|^{2}-\frac{|a|^{2}}{\left(|a|^{2} R^{2}-1\right)^{2}}-\frac{|a|^{2}}{|a|^{2} R^{2}-1} \\
& \Longleftrightarrow\left|z-\frac{a}{|a|^{2} R^{2}-1}\right|^{2} \geq \frac{|a|^{4} R^{2}}{\left(|a|^{2} R^{2}-1\right)^{2}} \\
& \Longleftrightarrow\left|z-\frac{a}{|a|^{2} R^{2}-1}\right| \geq \frac{|a|^{2} R}{|a|^{2} R^{2}-1} .
\end{aligned}
$$

\section{References}

[1] Anderson, N., Saff, E. B., and Varga, R. S. On the Eneström-Kakeya theorem and its sharpness. Linear Algebra Appl., 28 (1979), 5-16.

[2] Anderson, N., Saff, E. B., and Varga, R. S. An extension of the Eneström-Kakeya theorem and its sharpness. SIAM J. Math. Anal., 12 (1981), 10-22.

[3] Aziz, A. and Mohammad, Q.G. On the zeros of a certain class of polynomials and related analytic functions. J. Math. Anal. Appl., 75 (1980), 495-502.

[4] Aziz, A. and Zargar, B.A. Some extensions of Eneström-Kakeya theorem. Glasnik Matematički, 31 (1996), 239-244. 
[5] Cauchy, A.L. Sur la résolution des équations numériques et sur la théorie de l'élimination. Exercices de Mathématiques, Quatrième Année, p.65-128. de Bure frères, Paris, 1829. Also in: Oeuvres Complètes, Série 2, Tome 9, 86-161. GauthiersVillars et fils, Paris, 1891.

[6] Eneström, G. Härledning af an allmän formel for antalet pensionärer vid en godtycklig tidpunkt förefinnas inom en sluten pensionskassa. Öfversigt af Kungl. VetenskapAkademiens Förhandlinger (Stockholm), 50 (1893), 405-415.

[7] Eneström, G. Remarque sur un théorème relatif aux racines de l'équation $a_{n} x^{n}+\ldots+a_{0}$ où tous les coefficients sont réels et positifs. Tôhoku Math. J., 18 (1920), 34-36. Translation of reference [6].

[8] Gardner, R.B. and Govil, N.K. Enestrm-Kakeya theorem and some of its generalizations. Current topics in pure and computational complex analysis, 171-199. Trends Math., Birkhuser/Springer, New Delhi, 2014.

[9] Hurwitz, A. Ueber einen Satz des Herrn Kakeya. Tôhoku Math. J., 4 (1913-14), 89-93; Math. Werke, Vol. 2, 626-631.

[10] Kakeya, S. On the limits of the roots of an algebraic equation with positive coefficients. Tôhoku Math. J., 2 (1912), 140-142.

[11] Marden, M. Geometry of polynomials. Second edition. Mathematical Surveys, No. 3, American Mathematical Society, Providence, R.I., 1966.

[12] Melman, A. The twin of a theorem by Cauchy. Amer. Math. Monthly, 120 (2013), 164-168.

[13] Melman, A. Geometric aspects of Pellet's and related theorems. Rocky Mountain J. Math., 45 (2015), 603-621.

[14] Melman, A. Improved Cauchy radius for scalar and matrix polynomials. Proc. Amer. Math. Soc., 146 (2018), 613-624.

[15] Milovanović, G. V., Mitrinović, D. S., and Rassias, Th. M. Topics in polynomials: extremal problems, inequalities, zeros. World Scientific Publishing Co., Inc., River Edge, NJ, 1994.

[16] Rahman, Q.I., and Schmeisser, G. Analytic Theory of Polynomials London Mathematical Society Monographs. New Series, 26. The Clarendon Press, Oxford University Press, Oxford, 2002. 